\title{
Biochemical Markers in Perinatal Asphyxia
}

\author{
Naithani $\mathbf{M}^{1}$, Simalti $\mathbf{A K}^{2}$ \\ ${ }^{1}$ Dr. Manisha Naithani, MBBS, MD, Associate Professor, Department of Biochemistry, Sri Guru Ram Rai Institute of \\ Medical Sciences, Deheradun, India, ${ }^{2}$ Dr. Ashish Kumar Simalti, MBBS, MD. Graded Specialist in Paediatrics, Military \\ Hospital, Agra.
}

Address for correspondence: Dr. AK Simalti, E-mail: ashishsimalti@rediffmail.com

\begin{abstract}
Early assessment of the severity of an acute cerebral lesion secondary to hypoxia-ischemia or other pathologic conditions may provide a very useful basis for preventive or therapeutic decisions in pediatric patients. In the present review, we discuss the diagnostic and prognostic value of a series of biochemical parameters, with special reference to the diagnosis of neonatal HIE. Currently many specific biochemical markers of brain injury are being investigated to assess regional brain damage after perinatal asphyxia in neonates of which serum protein S-100 $\beta$, brain-specific creatine kinase, neuron-specific enolase, IL6 and urinary uric acid levels appear promising in identifying patients with a risk of developing hypoxic-ischemic encephalopathy. Whether detection of elevated serum concentrations of these proteins reflects long-term neurodevelopmental impairment remains to be investigated.
\end{abstract}

Key words: S-100, Brain specific creatine kinase, neuron specific enolase, IL6, urinary uric acid, hypoxic ischaemic cerebral injury.

\section{Introduction}

$\mathrm{H}^{\mathrm{s}}$ ypoxic-ischemic cerebral injury that occurs during the perinatal period is one of the most commonly recognized causes of severe, long-term neurological deficits in children. Hypoxic-ischemic encephalopathy (HIE) of the newborn occurs with the incidence of $1-4 / 1000^{1}$. Between $20 \%$ and $50 \%$ of newborn infants affected by perinatal brain injury die during the newborn period, and 25-60\% of the survivors suffer from permanent neurodevelopmental handicaps, including cerebral palsy, seizures, mental retardation, and learning disabilities ${ }^{2,3}$. Early assessment of the severity of an acute cerebral lesion secondary to hypoxiaischemia or other pathologic conditions may provide a very useful basis for preventive or therapeutic decisions in pediatric patients. Some authors have suggested that biochemical indicators may be more effective than the results of clinical evaluation, Apgar score, $\mathrm{pH}$ in cord blood, electroencephalographic and neuroimaging data $^{4}$. Among the earliest biochemical markers of brain hypoxia in the CSF and/or plasma studied were lactate which accumulates in hypoxic cells due to glycolysis ${ }^{5}$. Among enzymes released from the cells during brain hypoxia, the first studied were LDH with isoenzymes, creatine-kinase (particularly its brain-specific isoenzyme CK-BB) and adenylate kinase. In the present review, we discuss the diagnostic and prognostic value of a series of biochemical parameters, with special reference to the diagnosis of neonatal HIE.

\section{S100B}

The term S100B refers to members of a multigenic family of calcium-modulated proteins (S100 proteins), mostly of low molecular mass (10,000 Da), At present, at least 20 proteins have been identified as belonging to the $\mathrm{S} 100$ protein family, the members of which are characterized by the presence of a pair of so-called EF-hand (i.e., helix-loop-helix) calcium-binding motifs ${ }^{6}$. Interestingly, the S100 proteins are highly conserved in amino acid composition among vertebrate species, and S100-like proteins have also been immunologically detected in planarians and spinach leaves S100B, a homodimer of a subunit ( $B$ subunit) that constitutes the bulk of the fraction originally isolated from brain extracts, was regarded for more than a decade as specific to the nervous system ${ }^{7}$. In the nervous system, 
the protein appears to be most abundant in glial cells, although its presence in neuronal subpopulations has also been reported ${ }^{9}$. The biological role of this protein within the cell populations that contain it has not been completely elucidated, although many hypotheses have been formulated, including the inhibition of protein phosphorylation, inhibition of cytoskeletal constituent assembly, stimulation of enzyme activities, and interaction with transcription factors ${ }^{9}$. An extracellular biological role has also been hypothesized for S100B, which secreted by astrocytes as a cytokine could have a neurotrophic effect during both development and nerve regeneration at physiologic (nanomolar) concentrations, but at high (micromolar) concentrations could be neurotoxic, participating in the pathophysiology of neurodegenerative disorders ${ }^{10,11}$. In this respect, it could be relevant that $\mathrm{S} 100 \mathrm{~B}$ is coded on the long arm of chromosome 21 (21q22.3), which is also involved in the translocation that causes Down syndrome ${ }^{12}$. An increase in gene expression for the protein has also been related to the neurodegenerative processes associated with both Down syndrome and Alzheimer disease, on the basis of findings indicating that $ß$-amyloid stimulates the synthesis of S100b, whereas ß-amyloid precursor protein increases in cultures exposed to $\mathrm{S}_{100 \mathrm{~B}^{13}}$. Longitudinal S100B protein measurements in urine soon after birth are a useful tool to identify which asphyxiated infants are at risk of long-term neurologic sequelae. In a study by Diego Gazzolo, et al S100B protein levels were significantly higher in samples collected at all monitoring times from new borns with abnormal neurologic findings on follow-up. An S100B concentration cutoff of $0.28 \mu \mathrm{g} / \mathrm{L}$ at first urination had a sensitivity of $100 \%$ and a specificity of $87.3 \%$ for predicting the development of abnormal neurologic findings on follow-up. The sensitivity and specificity of measurements obtained between 12 and 72 hours were up to $100 \%$ and $98.2 \%$, respectively ${ }^{14}$.

\section{Neuron Specific Enolase}

Neuron-Specific Enolase (NSE), a homodimer of the gamma form of enolase, is localized in the cytoplasm of neurons and cells of neuronal origin. NSE is an enzyme which catalyzes the conversion of 2-phosphoglycerate to phosphoenolpyruvate in the glycolytic pathway, and also the reverse reaction in gluconeogenesis ${ }^{15}$. Perhaps not surprisingly since neurons require a great deal of energy they are very rich in glycolytic enzymes such a GAPDH and NSE. Its presence in cerebrospinal fluid and blood is attributed to cell destruction. Serum NSE level is a marker for neuronal damage after traumatic brain injury, tumor, and neurodegenerative diseases ${ }^{16}$.

In a study by Giuseppe, Distefano et al Protein S-100 (PS-100) and Neuron Specific Enolase (NSE) serum levels were studied serially during the perinatal period in preterm neonates with perinatal asphyxia as markers of glial and neuronal damage respectively. The results of this study showed that neonates with severe asphyxia at any time had significantly more elevated mean serum levels of both markers compared to the group with mild asphyxia and to the control group $(p<0.05)$. The values of control group were also significantly lower in comparison with that of mild asphyxia. In neonates with severe asphyxia, NSE values decreased constantly from birth to the seventh day of life, while PS-100 showed a different pattern increasing progressively between $3 \mathrm{~h}$ and 7 days. In neonates with mild asphyxia serum values of both markers showed decreasing levels through the study period ${ }^{17}$.

Tekgul and colleagues also reported that the levels of NSE in cerebrospinal fluid (CSF) were significantly higher in cases of moderate to severe HIE vis-à-vis mildly affected infants. However, they found that though the CSF levels of NSE correlated relatively strongly with the degree of HIE, the serum NSE levels did not show significant correlation with the same ${ }^{18}$.

Also, Verdú Pérez A, et al found that, the presence of elevated NSE values in blood after perinatal asphyxia can be a sensitive indicator of conspicuous brain damage. The combined information provided by the severity of the encephalopathy together with the blood NSE values, has high predictive value for neurological outcome. NSE levels in the group of neonates who died or developed neurological sequelae were significantly higher than NSE values in the group of neonates who were neurologically normal at follow-up. There were not differences between NSE values in the group of neonates who developed neurologically normal and the control group. Sensitivity and specificity of blood NSE as predictor of poor outcome were, respectively, $100 \%$ and $78 \%$. The combined specificity for blood NSE together with a moderate/severe encephalopathy was $95 \%{ }^{19}$.

In a similar study by $E$ Thornberg NSE was significantly increased in the CSF of infants with HIE (median value $25.4 \mathrm{pug} / \mathrm{l}$ ) compared with control infants (10.0 pug/l). Infants with the highest concentrations $\operatorname{died}^{20}$. So we can say that blood levels of NSE may or may not be reliable indicator in view of contradicting reports of studies but CSF levels are reliable predictors.

\section{IL6}

Experimental studies suggest that cytokinemediated inflammatory reactions are important in the cascade leading to hypoxic ischemic brain injury. In their study Tekgul et al found that the levels of IL-6 in 
both serum and CSF were significantly higher in infants with moderate to severe HIE than in the mildly affected infants $^{18}$. IL- 6 in CSF best predicted the outcome at age 2 years. A cutoff value of $25.9 \mathrm{pg} / \mathrm{mL}$ was $100 \%$ sensitive and $79 \%$ specific for an adverse outcome, and had positive and negative predictive values of $100 \%$ and $86 \%$, respectively. Sävman, Karin in a study measured the content of pro- and anti -inflammatory cytokines in CSF of asphyxiated and control infants. The concentrations of IL-1 $\beta$, IL-8, IL-10, tumor necrosis factor (TNF)- $\alpha$, and granulocyte/monocyte colony-stimulating factor (GMCSF) were determined. The concentration of IL-6 was higher in asphyxiated than in control. There was also a significant relationship between IL- 6 and the degree of HIE, and the final outcome ${ }^{21}$. Xanthou also reported a rise in serum levels of IL-6, IL-1 $\beta$ and sICAM-1 in perinatally asphyxiated and infected neonates ${ }^{22}$. Martín Ancel and colleagues concluded in their study that the magnitude of IL-6 response in the CSF after perinatal asphyxia is related to the severity of neonatal HIE, to brain damage, and to the neurological outcome ${ }^{23}$. Various other centres have also reported comparable results to suggest that IL-6 might play a role in neonatal hypoxic-ischemic brain damage ${ }^{24,25}$.

\section{Urinary Uric Acid}

Tissue hypoxia is a recognized cause of adenine nucleotide depletion, which is accompanied by the appearance of intermediate catabolites. These intermediates are used as metabolic markers of ATP depletion in tissues. Oxypurines (hypoxanthine and xanthine) are the final products of ATP degradation followed by uric acid formation ${ }^{26,27}$. Under normal conditions, about $90 \%$ of the hypoxanthine is reconverted to purine nucleotides by the action of HGPRT, restoring the ATP pool. Hypoxanthine is also converted to xanthine without the production of free radicals by the action of xanthine dehydrogenase, using nicotinamide adenine dinucleotide as substrate. During hypoxia, cellular ATP concentrations drop and there is accelerated degradation of adenosine monophosphate (AMP) to hypoxanthine ${ }^{28}$. Several experimental studies have indicated some xanthine oxidase activity in cerebral capillaries, and raised uric acid levels after periods of hypoxia ${ }^{29}$.

Perlman and Risser determined serum uric acid levels over the first 2 days post-partum in premature neonates at risk for intraventricular hemorrhage or periventricular leukomalacia, and found that levels were significantly raised in neonates who subsequently developed either of these complications ${ }^{30}$.

David Bader, David Gozal et al conducted a study to asses the validity of urinary uric acid as a marker of the degree of perinatal asphyxia. The ratio of urinary uric acid to creatinine (UA/Cr) in urine specimens obtained after birth were significantly higher in asphyxiated infants who were identified by an Apgar scores $\leq 5$ at $5 \mathrm{~min}$ and/ or an umbilical cord blood $\mathrm{pH} 7.2$, and a base deficit $12 \mathrm{meq} / 1$. Also, within the perinatal asphyxia group, a significant correlation was found between the $\mathrm{UA} / \mathrm{Cr}$ ratio and the asphyxia score ${ }^{31}$.

During reoxygenation after hypoxia, hypoxanthine is oxidized by the action of xanthine oxidase, forming xanthine. This reaction generates free radicals (superoxide anion, hydrogen peroxide, hydroxyl radical), which may in turn cause tissue damage. The xanthine-oxidase inhibitor allopurinol (ALLO) reduces free radical formation, thereby limiting the amount of hypoxia-reperfusion damage. Animal and human studies suggest that administration of ALLO immediately prior to delivery in case of suspected foetal asphyxia might reduce hypoxic-ischaemic encephalopathy. Kaandorp et al have proposed a study on effect of antenatal injection of allopurinol ${ }^{32}$.

Chen $\mathrm{HJ}$, et al studied the validity of urinary uric acid as a marker of perinatal asphyxia in term and premature infants. The urinary $\mathrm{UA} / \mathrm{Cr}$ was significantly higher in term and preterm infants with perinatal asphyxia than in their unasphyxiated counterparts. The urinary UA/Cr in premature infants was significantly higher than in term infants. When the urinary UA/Cr was $>0.95$, perinatal asphyxia was identified with a sensitivity of $80 \%$ and a specificity of $71 \%$ in term infants. They concluded that the urinary UA/Cr may be used as an additional marker of perinatal asphyxia in term and premature infants. In comparison with other markers it is a simple, quick, and inexpensive way to detect hypoxic episodes in a neonatal intensive care unit within 24 hours after birth ${ }^{33}$.

\section{Creatinine Kinase}

Creatinine kinase BB (CK), a key enzyme in the energy metabolism of cells with intermittently high and fluctuating energy requirement (e.g. skeletal and cardiac muscle, neural tissues like brain and retina) catalyzes the reversible transfer of the phosphoryl group from phosphocreatine to adenosine diphosphate, to regenerate adenosine triphosphate (ATP). The distinct isoenzyme-specific localization of $\mathrm{CK}$ isoenzymes in brain suggests an important function for $\mathrm{CK}$ in brain energy and points to adaptation of the $\mathrm{CK}$ system to special energy requirements of different neuronal and glial cell types. The determination of CK and its histospecific isoenzymes in serum is a standard technique for evaluating disorders that affect the 
myocardium, the skeletal muscle and the central nervous system (CNS). Hence, serum and CSF brain derived $\mathrm{CK}$ (CK-BB) isoenzyme determination in neonates, has been suggested to be of value for predicting the degree of neuronal damage and even developmental prognosis.

V J Ruth studied serum CK-BB activity on the first day of life in acutely asphyxiated infants, infants born after high risk pregnancies (pre-eclampsia/ intrauterine growth retardation, or both), and very low birth weight infants. Neuro-developmental evaluation was carried out at 2.2-2.5 years. They showed that infants who died with brain injury had significantly higher CK-BB activity than infants with normal outcomes. CK-BB in infants with cerebral palsy and mild motor impairment were similar to controls ${ }^{34}$.

Cuestas observed that in healthy neonate's serum CK-BB activity declines rapidly after birth, reaching a stable value after $6 \mathrm{~h}-15$ days, whereas neonates with severe asphyxia and neurologic damage showed a rapid increase in CK-BB concentration ${ }^{35}$.

Walsh and colleagues monitored CK-BB activity in cord blood over 6-80h post-partum, in normal and severely asphyxiated neonates, finding that CK-BB activities 2.5 standard deviations higher than the control-group correctly predicted subsequent neurologic abnormality in 17 (77\%) of the 22 asphyxiated neonates, and in $11(92 \%)$ of the 12 asphyxiated neonates who survived the neonatal period. These authors concluded that serum CK-BB activity determined in cord blood between 6 and $12 \mathrm{~h}$ post-partum is an effective predictor of neurological prognosis following severe neonatal asphyxia ${ }^{36}$.

Other studies have looked at CK-BB in CSF. In their independent trials, Dalens ${ }^{37}$ and Talvik ${ }^{38}$ observed that CSF CK-BB levels determined on day three of life was an effective predictor of neurologic anomalies detected at 12 months of age. Nagdyman Nicole et al in a study examined CK-BB, protein S-100, and neuron-specific enolase in cord blood and 2, 6, 12, and $24 \mathrm{~h}$ after birth in 29 asphyxiated and 20 control infants. A combination of serum protein S-100 and CK-BB 2 hours after birth had the highest predictive value and specificity of predicting moderate and severe HIE. Cord blood $\mathrm{pH}$ and cord blood base deficit increased the predictive values of protein S-100 and CK-BB ${ }^{39}$

\section{Glial Fibrillary Acidic Protein (GFAP)}

Glial fibrillary acidic protein (GFAP) is the structural protein of intermediate filaments in astroglia. In the CSF, it is typically present at very low concentration.
Acute CNS insults may lead to very high concentrations in CSF, probably because of the disintegration of astroglial cells, while moderate increases are observed in other clinical conditions such as Alzheimer's disease, dementia secondary to multiple infarct, and infantile autism. GFAP has extensively been used as a marker of gliosis in neuropathology. HIE after perinatal asphyxia is a condition in which levels of GFAP might be elevated if brain cell damage occurs. In animal studies, the core of the hippocampus has been shown to be surrounded by a well-developed network of GFAP-positive cells providing an apparent protective shield. That shield was less developed in cultures from asphyxia-exposed animals ${ }^{40}$. In a study of twenty-two asphyxiated infants, Blennow et al. observed that the extremely high concentrations of GFAP and others brain-specific proteins in the CSF were mainly found in the group of babies that died. They concluded that the cerebrospinal fluid levels of GFAP might be an important adjunct in the neonatal assessment of infants subject to perinatal asphyxia, and together with other neuronal or glial proteins, it might also help in defining temporal relationships in asphyxia $^{41,42}$

\section{Conclusion}

The diagnosis of perinatal insults currently relies on adequate documentation of general medical and obstetric factors and on radiologic and laboratory assessments. To facilitate therapeutic strategies, early identification of the infant at highest risk for developing seizures secondary to hypoxia ischemia or asphyxia is critical, particularly if novel but potentially toxic therapies currently under experimental investigation become available for clinical use. Currently many specific biochemical markers of brain injury are being investigated to assess regional brain damage after perinatal asphyxia in neonatesof which serum protein S-100 $\beta$, brain-specific creatine kinase, neuron-specific enolase, IL6 and urinary uric acid levels appear promising in identifying patients with a risk of developing hypoxic-ischemic encephalopathy. Whether detection of elevated serum concentrations of these proteins reflects long-term neurodevelopmental impairment remains to be investigated.

\section{References}

1. Vannucci RC. Hypoxic-ischemic encephalopathy. Am J Perinatol 2000;17:113-20.

2. Derganc M, Osredkar D. Hypoxic-ischemic brain injury in the neonatal period. Zdrav Vestn 2008;77:51-8.

3. Miller SP, Ramaswamy V, Michelson D, et al. Patterns of brain injury in term neonatal encephalopathy. J Pediatr 2005;146:453-60. 
4. Vilanova Juanola JM, Figueras Aloy J, Palomeque Rico A, Roldán Ros A, Jiménez González R. Marcadores bioquímicos de hipoxia-isquemia cerebral. Acta Pediatr Esp 1994;52:422-27 (in Spanish).

5. Huang CC, Wang ST, Chang YC, Lin KP, Wu PL. Measurement of the urinary lactate:creatinine ratio for the early identification of newborn infants at risk for hypoxic-ischemic encephalopathy. N Engl J Med 1999;341:328-35.

6. Michetti1a F, Gazzolo D. S100B Protein in Biological Fluids: A Tool for Perinatal Medicine. Clinical Chemistry 2002;48:2097-104.

7. Kawasaki H, Nakayama $\mathrm{S}$, Kretsinger $\mathrm{RH}$. Classification and evolution of EF-hand proteins. Biometals 1998;11:277-95.

8. Kretsinger RH, Nockolds CE. Carp muscle calciumbinding protein: II Structure determination and general description. J Biol Chem 1973;248:331326.

9. Donato R. S100: a multigenic family of calciummodulated proteins of the EF-hand type with intracellular and extracellular functional roles. Int $J$ Biochem Cell Biol 2001;33:637-68.

10. Heizmann CW. Calcium-binding proteins in the central nervous system. Neurochem Res 1999;24:1097-1100.

11. Scotto C, Deloulme JC, Rousseau D, Chambaz E, Baudier J. Calcium and S100B regulation of p53dependent cell growth arrest and apoptosis. Mol Cell Biol 1998;18:4272-81.

12. Allore R, O'Hanlon D, Price R, Neilson K, Willard $H F$, Cox DR, et al. Gene encoding the $ß$ subunit of S100 protein is on chromosome 21: implications for Down's syndrome. Science 1988;239:1311-3.

13. Pena LA, Brecher CW, Marshak DR. Amyloid regulates gene expression of glial trophic substance $\mathrm{S} 100 ß$ in C6 glioma and primary astrocyte cultures. Mol Brain Res 1995;34:118-26.

14. Gazzolo D, Marinoni E, lorio R D, Bruschettini M, Kornacka M, Lituania Met al. Measurement of Urinary S100B Protein Concentrations for the Early Identification of Brain Damage in Asphyxiated Full-term Infant. Arch Pediatr Adolesc Med 2003;157:1163-8.

15. Çeltik C K, Acuna B, Öner N, Ö Pala. Neuronspecific enolase as a marker of the severity and outcome of hypoxic ischemic encephalopathy. Brain and Development 2004; 26 (6) : 398-402.

16. Begaz, T, Kyriacou, D N, Segal J, and Bazarian, J. Serum biochemical markers for post-concussion syndrome in patients with mild traumatic brain injury. J. Neurotrauma 2006;23:1201-10.

17. G Distefano, S Curreri, P Betta, Giovanni L V et al. Perinatal Asphyxia in Preterm Neonates Leads to Serum Changes in Protein S-100 and Neuron Specific Enolase. Current Neurovascular Research 2009;6(2):110-6.

18. Tekgul $\mathrm{H}$, Yalaz M, Kutukculer $\mathrm{N}$ et al. Value of biochemical markers for outcome in term infants with asphyxia. Pediatr Neurol 2004;31:326-32.

19. Verdú Pérez A, Falero MP, Arroyos A, Estévez F, Félix $V$, López $Y$ et al. Neuronal specific enolase in newborns with perinatal asphyxia. Neurology 2001;16-30.

20. Thornberg E, Thiringer K, Hagberg H, Kjellmer I. Neuron specific enolase in asphyxiated newborns: association with encephalopathy and cerebral function monitor trace. Arch Dis Child 1995;72:3942.

21. Sävman K, Blennow M, Gustafson K, Tarkowski E, Hagberg H. Cytokine response in cerebrospinal fluid after birth asphyxia. Pediatr Res 1998; 43: 746-51.

22. Xanthou M, Fotopoulos S, Mouchtouri A, Lipsou N, Zika I, J. Sarafidou. Inflammatory mediators in perinatal asphyxia and infection. Acta Paediatrica 2002; 91(9):92-7.

23. Martín-Ancel A, García-Alix A, Pascual-Salcedo D, Cabañas F, Valcarce M, Quero J. Interleukin-6 in the cerebrospinal fluid after perinatal asphyxia is related to early and late neurological manifestations. Pediatrics 1997;100:789-94.

24. Aly $\mathrm{H}$, Khashaba MT, El-Ayouty M, El-Sayed O, Hasanein BM. IL-1beta, IL-6 and TNF-alpha and outcomes of neonatal hypoxic ischemic encephalopathy. Brain Dev 2006;28:178-82.

25. Nelson KB, Dambrosia JM, Grether JK, Phillips TM. Neonatal cytokines and coagulation factors in children with cerebral palsy. Ann Neurol 1998;44:665-75.

26. Fox IH. Metabolic basis for disorders of purine nucleotide degradation. Metabolism 1981;30:61634. 
27. Hällgren R, Niklasson F, Terent A, Akerblom A, Widerlöv $E$. Oxypurines in cerebrospinal fluid as indices of disturbed brain metabolism. A clinical study of ischemic brain diseases. Stroke 1983;14:382-8.

28. Spector R. Hypoxanthine transport and metabolism in the central nervous system. I Neurochem 1988;50:969-78.

29. Betz AL. Identification of hypoxanthine transport and xanthine oxidase activity in brain capillaries. $J$ Neurochem 1985;44:574-79.

30. Perlman JM, Risser R. Relationship of uric acid concentrations and severe intraventricular hemorrhage/leukomalacia in the premature infant. J Pediatr 1998;132:436-39.

31. Bader D, Gozal1 D, Weinger-Abend M, Berger A, Lanir A. Neonatal urinary uric acid/ceratinine ratio as an additional marker of perinatal asphyxia. European Journal of Pediatrics 1995;154:324-32.

32. Kaandorp $\mathrm{J} J$, Benders $M J$, Rademaker $C$, Torrance H L, Oudijk M A, Haan T R, et al.Antenatal allopurinol for reduction of birth asphyxia induced brain damage (ALLO-Trial); a randomized double blind placebo controlled multicenter study. BMC Pregnancy and Childbirth 2010, 10: 2393-408.

33. Chen HJ, Yau KI, Tsai KS.Urinary uric acid/ creatinine ratio as an additional marker of perinatal asphyxia. J Formos Med Assoc 2000;99(10):771-4.

34. Ruth VJ. Prognostic value of creatine kinase BBisoenzyme in high risk newborn infants. Arch Dis Child 1989;64:563-8.
35. Cuestas RA Jr. Creatine kinase isoenzymes in highrisk infants. Pediatr Res 1980;14:935-38.

36. Walsh P, Jedeikin R, Ellis G, Primhak R, Makela SK. Assessment of neurologic outcome in asphyxiated term infants by use of serial CK-BB isoenzyme measurement. J Pediatr 1982; 101: 988-92

37. Dalens B, Viallard JL, Raynaud EJ, Dastugue B. Enzyme studies and neonatal brain damage. Acta Paediatr Scand 1981;70:743-49.

38. Talvik T, Haldre $S$, Sööt $A$, Hämarik $M$, Piirsoo A, Mikelsaar AV. Creatine kinase isoenzyme BB concentrations in cerebrospinal fluid in asphyxiated preterm neonates. Acta Paediatr 1995;84:1183-187

39. Nagdyman N, Grimmer I, Scholz T, Muller C, Obladen M. Predictive value of brain-specific proteins in serum for neurodevelopmental outcome after birth asphyxia. Pediatr Res 2003;54:270-75.

40. Morales $\mathrm{P}$, Reyes $\mathrm{P}$, Klawitter $\mathrm{V}$, Huaiquín $\mathrm{P}$, Bustamante D, Fiedler J, Herrera-Marschitz M.Effects of perinatal asphyxia on cell proliferation and neuronal phenotype evaluated with organotypic hippocampal cultures. Neuroscience 2005;135(2):421-31.

41. Blennow M, Hagberg $H$, Rosengren L. Glial fibrillary acidic protein in the cerebrospinal fluid: a possible indicator of prognosis in full-term asphyxiated newborn infants? Pediatr Res 1995; 37: 260-264.

42. Blennow $M$, Sävman $K$, Ilves $P$, Thoresen $M$, Rosengren $L$. Brain-specific proteins in the cerebrospinal fluid of severely asphyxiated newborn infants. Acta Paediatr 2001;90:1171-175.

\section{How to cite this article?}

Naithani M, Simalti AK. Biochemical Markers in Perinatal Asphyxia. J Nep Paedtr Soc 2011;31(2):151-156. 